\title{
Research on the Application of a Control Method for Multiple Precision Machining Equipment
}

\author{
Junfu Qiao and Jinqin Guo \\ Department of Automation, Taiyuan Institute of Technology University, Taiyuan \\ 030008, China \\ qiaojunfu@163.com
}

\begin{abstract}
Objective: to propose a high precision and high efficiency intelligent shaping grinding machine to improve the yield and the yield of solar energy. Methods: This paper presents a kind of intelligent control device with feedback and can automatically compensate. It mainly consists of the detection device, the compensation device, the main control device, the embedded control software. Process: combining the fuzzy predictive control model and ARM embedded system, it can transform the traditional machining grinding machine into the intelligent grinding machine. Results and analysis: the process of making the prism can save time and effort, and the accurate positioning of the embryo is accurate, and the precision of grinding is stable, and the processing time is shortened. Conclusion: the technology greatly improves the processing efficiency and processing quality, meets the demand of mass production, and further improve the competitiveness of China's solar prism products in the international market.Solar and other fields of high precision multi faceted pyramidal prism
\end{abstract}

Keyword: Intelligent forming grinding machine, Solar prism, Combined fuzzy predictive control model, ARM embedded system

\section{Introduction}

Solar glass, which is suitable for the solar energy equipment in the sun has a higher ordinary glass transmittance, or can be selected through glass. In solar glass, high transmittance is more widely used. Usually they are used to make a protective cover glass [1]. As far as the application is concerned, the main application area of solar energy is solar cell and flat plate solar collector.

At present, there is no high intelligence of the processing grinding machine of the solar prism in our country, the process is time-consuming and laborious, the positioning accuracy is low, the processing quality is poor, and the output and the qualified rate of the machine is very low[2-5]. Such efficiency is very difficult to meet the needs of the Chinese market, so it is urgent to develop high precision and high efficiency intelligent molding grinding machine to improve the yield and finished product rate, so it has some practical value and broad market prospect[6].

This project is developed by the "solar prism grinding machine control device" is a kind of intelligent control device with feedback, which can automatically compensate. It mainly consists of the detection device, the compensation device, the main control device, the embedded control software. The innovation of this project is the application of fuzzy predictive control model and ARM embedded system. In the use of this device, the traditional machining grinding machine can be transformed into intelligent grinding machine, which makes the assembly and saving time and effort, accurate positioning, stable and accurate, and greatly shorten the processing efficiency and processing quality. 


\section{Materials and Methods}

\subsection{Processing Equipment System}

The machining equipment, including grinding machining device and machining platform, can change the angle of the grinding head device, the rotating surface mechanism and the multiple cylinder, and on the same plane, through the tank wall extending to the grinding wheel, on the axis of the machine can be fitted by the clamping and fixing the workpiece on the corresponding belt teeth can be loaded on the matching corresponding to the adjacent tooth index [7].

By fixing the synchronization process on the polyhedron, the production efficiency is improved and the machining cost is reduced; on the indexing disc, the conversion of the polyhedron processing, indexing axis rotation and indexing disc drive synchronous rotation, processing surface conversion, the machining surface of the disc and the gap between the taper pin, thereby locking the indexing disc position, lock axis and indexing polyhedron processing position, relative worm lock torque, structure is simple, manufacturing cost is low, while eliminating the gap between the gear, the workpiece can be accurately locked position, positioning accuracy and processing accuracy, can meet the positioning requirements [8-9].

The servo motor (Figure 1) used in the system can be used to control the speed, the position accuracy is very accurate, and the voltage signal can be converted to the torque and speed to drive the control object. Servo motor rotor speed by the control signal input, and can respond quickly, in automatic control system that is used for the implementation of components, and has small electromechanical time constant, high linearity, initiating voltage characteristics, the received signal is converted into the motor shaft angular velocity or displacement output.

The grinding machine is mainly divided into three kinds of motion, main motion, grinding wheel of grinding spindle rotary motion; feed movement, including: table bed along the longitudinal guide rail straight reciprocating motion is grinding head along the sliding plate of a horizontal guide rail of transverse intermittent feeding; vertical feed motion, grinding head adjustment height position and control the grinding depth. In which the grinding machine of the physical map of the Figure 2, Figure 3, Figure 4. Table 1 describes the various parameters of the grinder equipment.

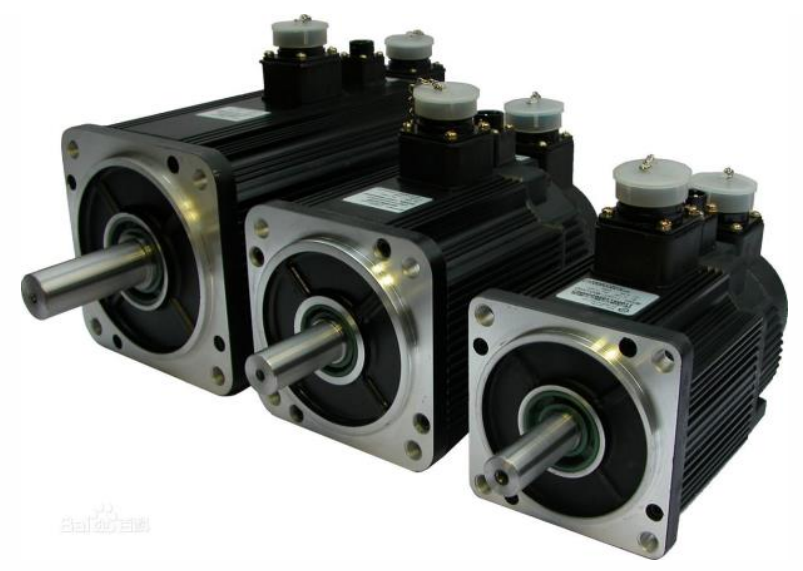

Figure 1. Servo Motor Physical Map 
The relationship between acceleration, velocity, displacement and time of the five-stage $\mathrm{S}$-curve acceleration and deceleration algorithm each motion stage is:

$$
\begin{aligned}
& a(t)=\left\{\begin{array}{cc}
J t & t \in\left[0, t_{1}\right] \\
2 J T-J t & t \in\left(t_{1}, t_{2}\right] \\
0 & t \in\left(t_{2}, t_{3}\right] \\
2 J T-J\left(t-T_{3}\right) & t \in\left(t_{3}, t_{4}\right] \\
-4 J T+J\left(t-T_{3}\right) & t \in\left(t_{4}, t_{5}\right]
\end{array}\right. \\
& v(t)=\left\{\begin{array}{cc}
v_{s}+\frac{1}{2} J^{2} & t \in\left[0, t_{1}\right] \\
v_{s}-J T^{2}+2 J T t-\frac{1}{2} J^{2} & t \in\left(t_{1}, t_{2}\right] \\
v_{c} & t \in\left(t_{2}, t_{3}\right] \\
v_{s}-J T^{2}+2 J T\left(t-T_{3}\right)-\frac{1}{2} J\left(t-T_{3}\right)^{2} & t \in\left(t_{3}, t_{4}\right] \\
v_{s}-4 J T^{2}+3 J T\left(t-T_{3}\right)-\frac{1}{2} J\left(t-T_{3}\right)^{2} & t \in\left(t_{4}, t_{5}\right]
\end{array}\right. \\
& s(t)=\left\{\begin{array}{cc}
\mathrm{v}_{\mathrm{s}} \mathrm{t}+\frac{1}{6} \mathrm{Jt}^{3} & \mathrm{t} \in\left[0, \mathrm{t}_{1}\right] \\
\frac{1}{3} \mathrm{JT}^{3}+\mathrm{v}_{\mathrm{s}} \mathrm{t}-\mathrm{JT}^{2} \mathrm{t}+\mathrm{JTt}^{2}-\frac{1}{6} \mathrm{Jt}^{2} & \mathrm{t} \in\left(\mathrm{t}_{1}, \mathrm{t}_{2}\right] \\
2 \mathrm{v}_{\mathrm{s}} \mathrm{T}+\mathrm{JT}^{3}-2 \mathrm{v}_{\mathrm{c}} \mathrm{T}+\mathrm{v}_{\mathrm{c}} \mathrm{t} & \mathrm{t} \in\left(\mathrm{t}_{2}, \mathrm{t}_{3}\right] \\
\mathrm{v}_{\mathrm{c}} \mathrm{T}_{\mathrm{c}}-\mathrm{v}_{\mathrm{s}} \mathrm{T}_{\mathrm{c}}+\frac{1}{3} \mathrm{JT}^{3}-\mathrm{JT}^{2} \mathrm{~T}_{3}+\frac{1}{6} \mathrm{JT}_{3}^{3}+\left(\mathrm{v}_{\mathrm{s}}-\mathrm{JT}^{2}\right) \mathrm{t}+\mathrm{JTt}^{2}-\frac{1}{6} \mathrm{Jt}^{3} & \mathrm{t} \in\left(\mathrm{t}_{3}, \mathrm{t}_{4}\right] \\
\frac{4}{3} \mathrm{JT}^{3}+\mathrm{v}_{\mathrm{c}} \mathrm{T}_{3}-\mathrm{v}_{\mathrm{s}} \mathrm{T}_{3}+\mathrm{v}_{\mathrm{s}} \mathrm{t}-\frac{1}{6} \mathrm{~J}\left(4 \mathrm{~T}+\mathrm{T}_{3}-\mathrm{t}\right)^{3} & \mathrm{t} \in\left(\mathrm{t}_{4}, \mathrm{t}_{5}\right]
\end{array}\right.
\end{aligned}
$$

From the above curves and deceleration control algorithm formula can be seen, as long as the variable $\mathrm{T}$ and $\mathrm{T} 3$ can be determined to construct the entire curve, and then determine the speed and displacement of each moment, compared with the traditional seven-stage S-curve acceleration and deceleration, the algorithm is relatively simple and easy to implement. In the box body corresponding to the sub axis coat of the box, the index plate fixed in the sub box on the axis of the cylinder, the cone pin coaxial connection in the lock cylinder of the connecting rod, the corner lock mechanism on the core axis through the box on a side wall, the drive shaft through the box on the other side of the box[10]. By setting the indexing box on the indexing axis, the connection between the indexing shaft and the angle locking mechanism and the angle mechanism are convenient. 


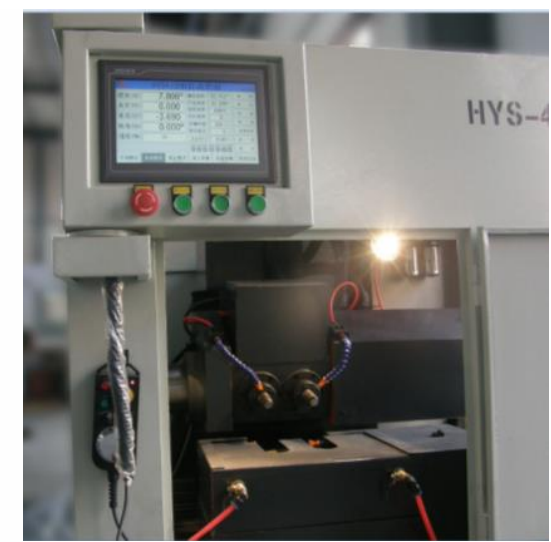

Figure 2. The Grinding Machine Equipment

The indexing box is arranged in a two or more than two or more than two axis, the indexing shaft extends to the indexing box, and a synchronous wheel is arranged on the external corresponding indexing axis, and the two synchronous wheels are connected to the driving motor by a synchronous belt[11]. By means of two axis fixed two polyhedron processing, the grinding disc is suitable to achieve the maximum efficiency of the production efficiency, save the processing cost, and the rotation of the two synchronous wheel is driven by an electric motor, which can simplify the structure and reduce the cost of manufacture and operation.

\section{Table 1. Parameters of Grinding Machine Equipment}

\begin{tabular}{|c|c|}
\hline Use power & three-phase $380 \mathrm{~V}$ \\
\hline Peak power & $8 \mathrm{KW}$ \\
\hline Average power & $4 \mathrm{KW}$ \\
\hline Angle precision & 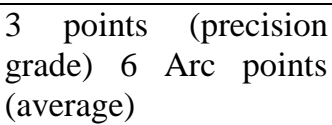 \\
\hline Dimension precision & $\begin{array}{l}0.02 \mathrm{~mm} \\
\text { grade) } \\
\text { (average) }\end{array}$ \\
\hline Spindle speed & $30000 \sim 0 \mathrm{rpm}$ \\
\hline The beating angle & less than $0.006 \mathrm{~mm}$ \\
\hline $\begin{array}{l}\text { The main axis of the } \\
\text { spindle }\end{array}$ & $0.01 \mathrm{~mm}$ \\
\hline Balancing spindle & less than $1 \mathrm{G}$ \\
\hline Standard & $\begin{array}{l}2 \text { grinding head } \\
\text { grinding head }\end{array}$ \\
\hline Controllable joint axis & 4 axis \\
\hline
\end{tabular}

Synchronous wheel side corresponding to the indexing box with positioning plate and between the synchronous wheels is arranged on the positioning plate and the indexing box and unclamping cylinder is arranged on the positioning plate, unclamping cylinder connecting rod extends coaxially to the pitch axis in and against connected to the clamping mechanism of the positioning rod[12]. The unclamping cylinder arranged on the indexing box, the unclamping cylinder and dividing box, indexing shaft rotate synchronously, so the replacement of indexing shaft work piece fixing rod (change of polyhedron) without indexing axis to the initial state, namely the indexing axis in any position can replace the work piece fixing rod, convenient use, improve processing efficiency. 


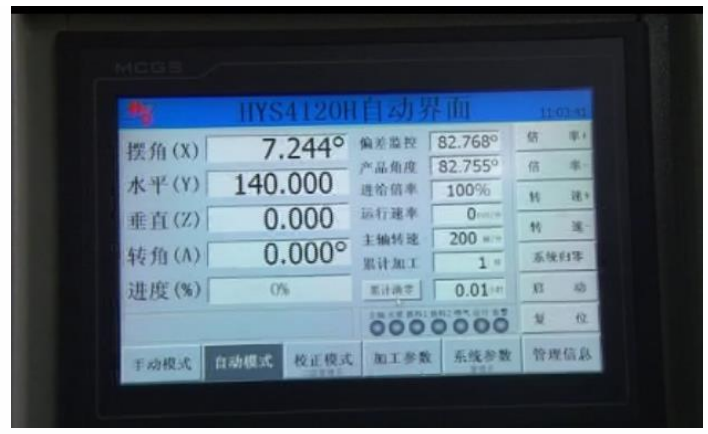

(a)

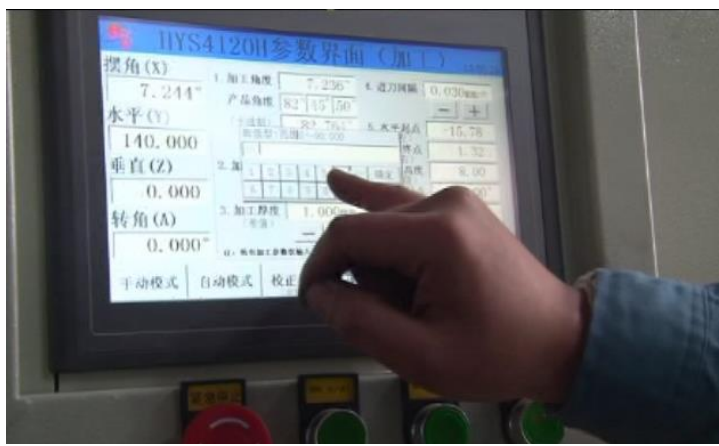

(b)

Figure 3. Grinder Touch Screen

The indexing disc is a disc shaped, and the dividing tooth is a straight tooth on the circular outer ring of the indexing disc, and the gap between the adjacent indexing teeth is a sector. Straight teeth, which are convenient for the precise coordination of the taper pin and the positioning accuracy, can meet the requirements of different angle of rotation.

The piston can also be used to drive compression, such as cylinder, screw rod, and the corner locking mechanism includes the core axis, which is arranged on the core axis of the box body, and the casing is provided with a corrugated sleeve, and the outer end of the corrugated sleeve is provided with a piston, and the piston is driven by a hydraulic drive system[13-14]. The core shaft is inserted into the side wall of the box body, and the casing is arranged between the core and the core, and the outer end of the corrugated sleeve is provided with a piston, and the box body needs to be locked and fixed on the core shaft, the corrugated sleeve is fixed in the box and the core shaft, the lock torque is large, the positioning accuracy is high, the hydraulic pressure is eliminated, and the elastic force of the bellows is more than the hydraulic pressure; through the sleeve of the inner side of the corrugated sleeve, the sleeve is set up, which can avoid the friction between the corrugated sleeve and core, reduce the core shaft wear, and can meet the requirement of the core shaft, save maintenance cost, save maintenance cost, and remove and change. 


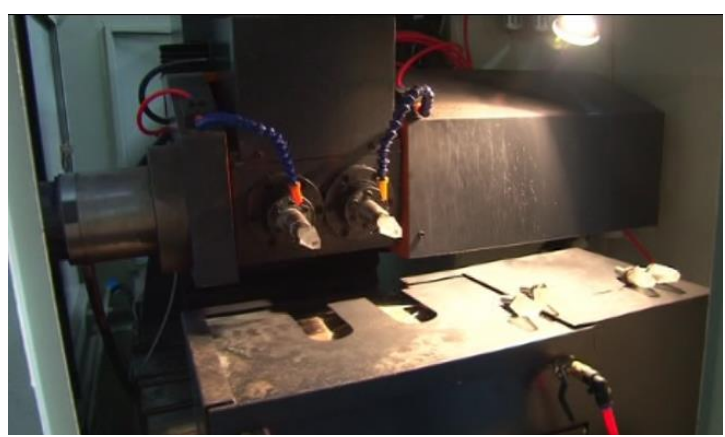

(a)

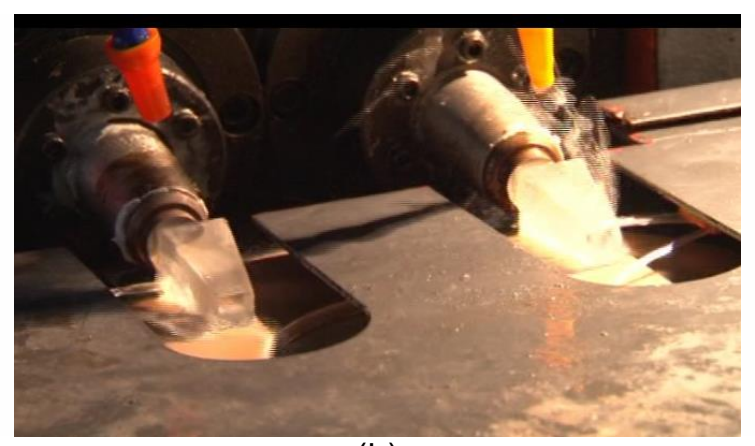

(b)

\section{Figure 4. The Head Physical Map}

The cone expansion mechanism includes a ring of inner ring and an outer ring of the inner ring of the inner ring of the inner ring of the inner ring of the outer ring, and the inner conical surface is arranged on the inner side of the outer ring with the external opening. Conical locking mechanism is a keyless connection device, it is through the high strength bolt tension axial inner and outer opening between the ring, thus, opening the core ring and the shaft between, the opening of the wear ring and sleeve produced huge hold force to achieve the wear-resistant sleeve and core axis keyless connection is simple in structure, high in accuracy, installation, adjustment, convenient disassembly, high strength, stable and reliable connection and the overload can protect connected components are not damaged and has the advantages of compact.

With adapter flange and butt on the adapter flange of the cylinder core shaft corresponding to the box body, core shaft after passing through the cylinder body and the adapter flange extends out the box body, corrugated sleeve and wear sleeve of the inner side of the top end of the on the outer end surfaces of the connecting flange[15]. Through the use of the core shaft in the case of the box axis on the outside of the docking port, and on the connecting flange of the cylinder, one is easy to connect and assembly, the two is to increase the core shaft and the contact area of the box to improve the positioning accuracy.

The angle mechanism comprises a drive shaft which is inserted into the side wall of the box body, and the outer end of the driving shaft is connected with the servo motor through a gear box, driven by the servo motor drive shaft driven by the rotation of the box, relative to the original worm gear rotation speed, high work efficiency.

The coaxial sleeve of the core shaft of the core shaft is provided with a position that can automatically identify the relative core axis of the box body, and the position grating is connected to the servo motor by the control system[16]. Through in the core shaft and the shaft end is provided with a servo motor is connected with 
the position of the grating, using servo motor drives the core rotate the shaft and box to achieve the polyhedral angle requirements, through the position of the grating can be detected at any time (machining polyhedral blank) box location and detection real-time feedback of information to the control system, and the control system to control the servo motor to rotate to re adjust the cabinet position, improve the polyhedral angle position accuracy, and to meet the processing requirements of high precision polyhedron. Position grating can be replaced by the encoder. Angle grating is installed on the corner mechanism to detect the angle of the work piece. The shaft of the driving wheel is provided with an encoder to measure the rotational speed of the grinding wheel, and the encoder and the control system are electrically connected. The hydraulic cylinder pressure sensor mounted on the tail end of the main shaft on the active detection, pressure cylinder work piece and lap in.

\subsection{Processing Equipment Calibration Method}

Calibration is mainly the absolute value (reference value) of the calibration; the accuracy has been completed by the grating. After the system is calibrated, the input parameters can be accurately reached to the desired product[17]. As a priority, the device is starting to work, but also need to coordinate the system coordinates, coordinate calibration including accuracy and mechanical characteristics correction, if the system coordinates for the first time correction, then the mechanical characteristics correction, if the system coordinates is not the first time correction, then into the line of accuracy. The mechanical characteristics are corrected as shown in Figure 5:

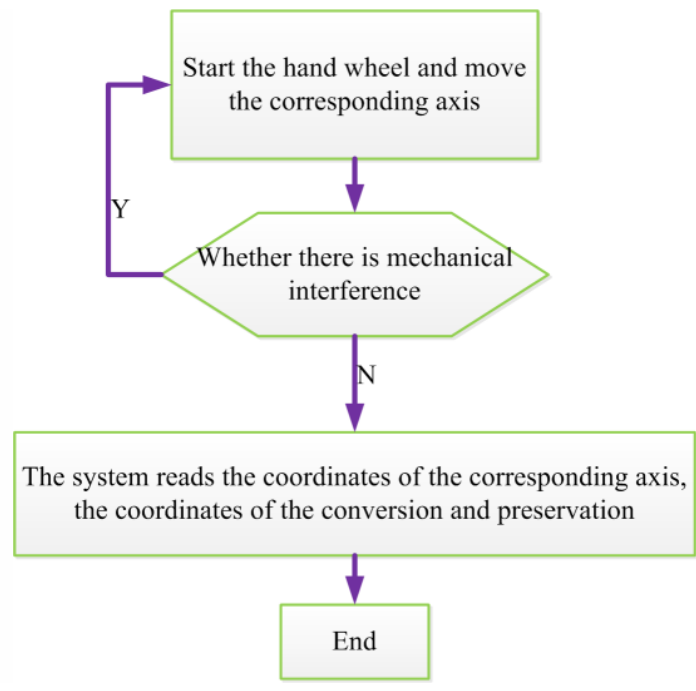

Figure 5. Mechanical Characteristic Correction

As a selection, the accuracy of the feature correction includes the angle back gap correction, the linear back gap correction, the processing angle correction and the work piece origin correction; the angle back gap correction is as follows: the angle of the motor is rotated to a point of view from the angle of the angle of the machine, and the angle of the machine is changed from the angle of the machine; the linear back gap correction is specific: continuously from the position of the work piece position, the motor is rotated to a position in one direction to the position of the position, and then reverse direction and the minimum unit drive servo motor to adjust the position of the work piece; processing angle correction as shown in Figure 6 , the origin of the work piece is shown in Figure 7. 




Figure 6. Processing Angle Correction

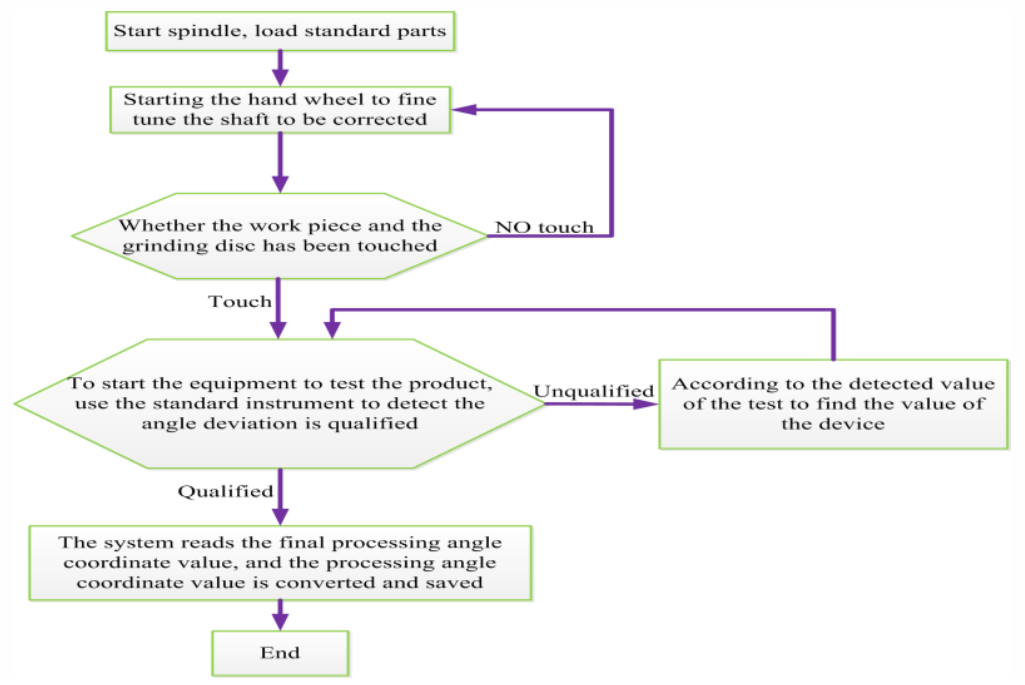

Figure 7. Work Piece Origin Correction

\subsection{Processing Equipment Control Method}

A control method for bull Precision indexing Processing equipment, the first judge whether the system is already zero, if it has been set to zero, then read the parameters, start multi task system, the angle, size and the spindle speed and pressure control, as shown in Figure 8. 


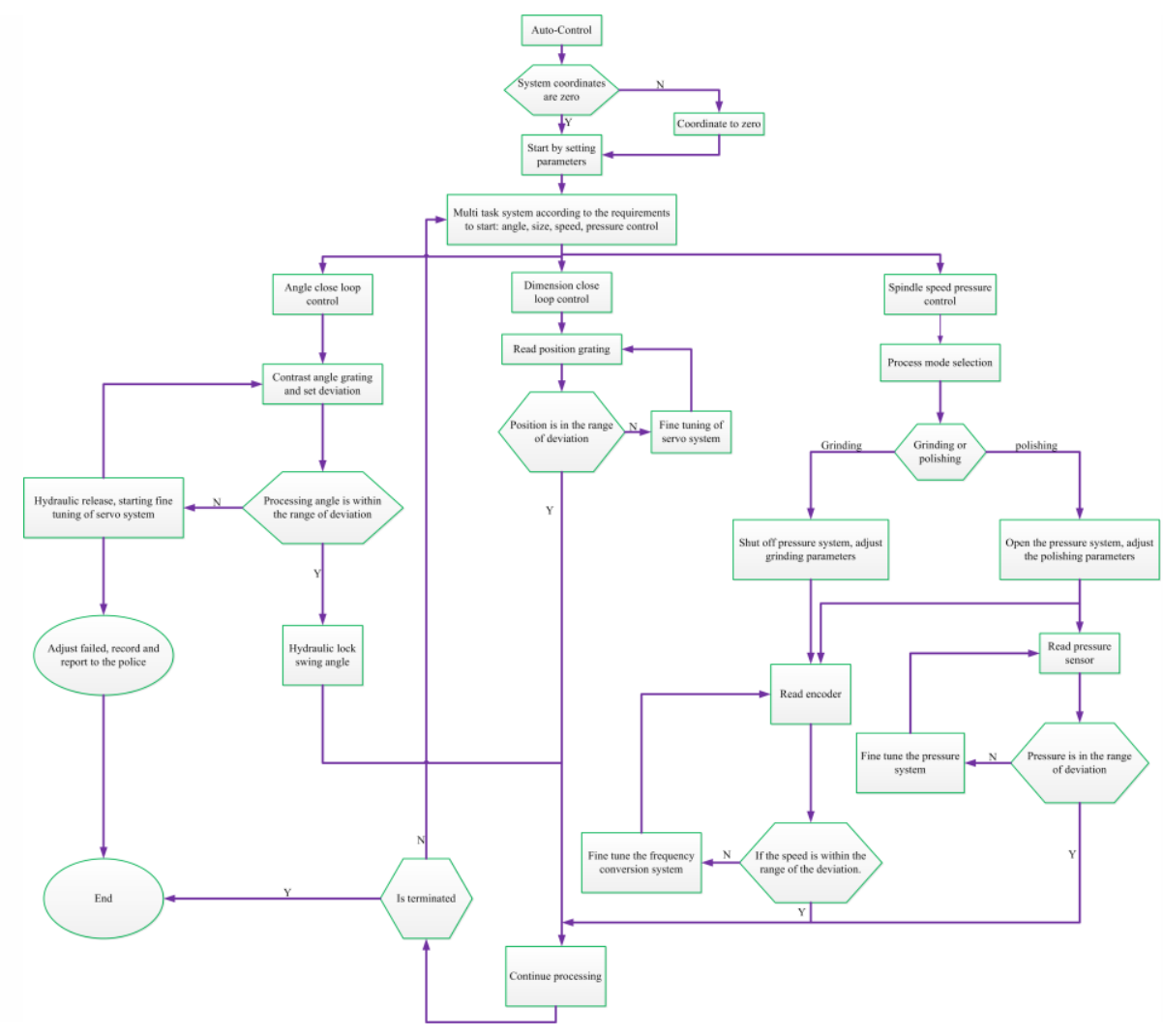

Figure 8. Processing Equipment Control Method Flow Chart

\section{An Example of the Five-Stage S-Curve Acceleration and Deceleration Algorithm}

In order to verify whether the actual operating frequency of the stepper motor is in line with the five section S curve, the photoelectric pulse encoder is used to match the AT91 SAM9261's capture mode TC module as the test system. The encoder is connected to the motor by the key and the stepping motor is used as a position detection device, and the detection pulse is transmitted as an input signal to the TIOB pin of the AT91 SAM9261 chip. In capture mode, when the TIOB pin detects a pulse rising edge, the TC counter value is loaded into the TIOB register (the counter is cleared at the same time) and the interrupt is triggered, and the pulse interval is obtained by reading the TIOB register value in the interrupt service routine.

The starting frequency of the motor control system is $1000 \mathrm{~Hz}$, the frequency is $1200 \mathrm{~Hz}$, the operating frequency is $6000 \mathrm{~Hz}$, the distance is $\mathrm{S} \mathrm{mm}$, Test system TC module clock frequency fCtrClk is $160 \mathrm{MHz}$, and from the motor start up to the stop process every $0.2 \mathrm{~s}$ sample read TIOB register value $\mathrm{N}$ is shown in Table 1(select acceleration part): 
Table 2. Record of Pulse Interval

\begin{tabular}{|c|c|c|c|}
\hline Moment & $\mathrm{N}$ & Moment & $\mathrm{N}$ \\
\hline 0.2 & 1600 & 2.4 & 473 \\
\hline 0.4 & 1568 & 2.6 & 430 \\
\hline 0.6 & 1481 & 2.8 & 398 \\
\hline 0.8 & 1355 & 3.0 & 373 \\
\hline 1.0 & 1212 & 3.2 & 355 \\
\hline 1.2 & 1066 & 3.4 & 341 \\
\hline 1.4 & 930 & 3.6 & 331 \\
\hline 1.6 & 808 & 3.8 & 325 \\
\hline 1.8 & 701 & 4.0 & 321 \\
\hline 2.0 & 610 & 4.2 & 320 \\
\hline 2.2 & 533 & & \\
\hline
\end{tabular}

The register value divided by the clock frequency is the actual time interval, the time interval is obtained by the reciprocal of the time:

$$
\mathrm{f}=\frac{\mathrm{f}_{\text {cricli }}}{\mathrm{N}}
$$

According to the type of each moment register record values into frequency values and the plotted curve, as shown in Figure 9:



Figure 9. Result of Pulse Test

From Figure 9 it can be seen that the acceleration process and the deceleration process of the stepping motor are in accordance with the law of $\mathrm{S}$ curve. The frequency of the pulse frequency switch is smooth and the motor runs stably. Moreover, the motor is accelerated to $5000 \mathrm{~Hz}$, which is based on the actual moving distance. By this control method of solar glass processing products was shown in Figure 10.

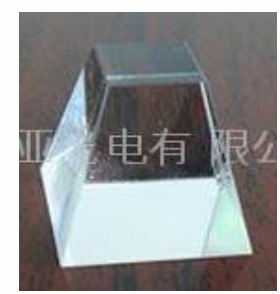

Figure 10. Solar Prism Glass Products 


\section{Conclusion}

This paper presents a control method for bull Precision indexing Processing equipment. This method first judges whether the system has zero or not. If the system has been set up, it can be used to control the angle, size and pressure. Through practice, the accuracy and efficiency of solar glass has been greatly improved by this method, to ensure the quality of products, this method deserves to be promoted.

\section{Acknowledgements}

Supported by Scientific and Technologial Innovation Programs of Higher Education Institutions in Shanxi (No.2013162).

\section{References}

[1] B. K. Yu, D. Vak and J. Jo, "Sequent spray deposition of secondary solvent for efficient polymer solar cells[J]", Macromolecular Research, vol. 23, no. 7, (2015), pp. 696-703.

[2] W. Yang, H. S. Duan and B. Bob, "Novel Solution Processing of High- Efficiency Earth- Abundant Cu2ZnSn (S, Se) 4 Solar Cells[J]”, Advanced Materials, vol. 24, no. 47, (2012), pp. 6323-6329.

[3] D. H. Wang, J. S. Moon and J. Seifter, "Sequential processing: control of nanomorphology in bulk heterojunction solar cells[J]", Nano letters, vol. 11, no. 8, , (2011), pp. 3163-3168.

[4] F. Dross, K. Baert and T. Bearda, "Crystalline thin- foil silicon solar cells: where crystalline quality meets thin- film processing[J]", Progress in Photovoltaics: Research and Applications, vol. 20, no. 6, (2012), pp. 770-784.

[5] A. Hinsch, W. Veurman and H. Brandt, "Worldwide first fully up- scaled fabrication of $60 \times 100 \mathrm{~cm} 2$ dye solar module prototypes[J]", Progress in Photovoltaics: Research and Applications, vol. 20, no. 6, (2012), pp. 698-710.

[6] H. J. Gwon, Y. Park and C. W. Moon, "Superhydrophobic and antireflective nanograss-coated glass for high performance solar cells[J]", Nano Research, vol. 7, no. 5, (2014), pp. 670-678.

[7] D. Bi, A. M. El-Zohry and A. Hagfeldt, "Improved morphology control using a modified two-step method for efficient perovskite solar cells[J]", ACS applied materials \& interfaces, vol. 6, no. 21, (2014), pp. 18751-18757.

[8] A. Ariyarit, K. Manabe and K. Fukada, "Semitransparent polymer-based solar cells via simple wet lamination process with $\mathrm{TiO} 2$ layer using automatic spray layer-by-layer method[J]", RSC Advances, vol. 5, no.65, (2015), pp. 52427-52435.

[9] Z. Liu, C. Liu and J. Ya, "Controlled synthesis of $\mathrm{ZnO}$ and $\mathrm{TiO} 2$ nanotubes by chemical method and their application in dye-sensitized solar cells[J]", Renewable Energy, vol. 36, vol. 4, (2011), pp. 1177-1181.

[10] H. J. Son, X. Wang and C. Prasittichai, "Glass-encapsulated light harvesters: More efficient dye-sensitized solar cells by deposition of self-aligned, conformal, and self-limited silica layers[J]", Journal of the American Chemical Society, vol. 134, no. 23, (2012), pp. 9537-9540.

[11] V. Zardetto, G. De Angelis and L. Vesce, "Formulations and processing of nanocrystalline TiO2 films for the different requirements of plastic, metal and glass dye solar cell applications[J]", Nanotechnology, vol. 24, no. 25, (2013), pp. 255401.

[12] M. S. Faber, K. Park and M. Cabán-Acevedo, "Earth-abundant cobalt pyrite (CoS2) thin film on glass as a robust, high-performance counter electrode for quantum dot-sensitized solar cells[J]", The Journal of Physical Chemistry Letters, vol. 4, no. 11, (2013), pp.1843-1849.

[13] J. E. Carlé, T. R. Andersen and M. Helgesen, "A laboratory scale approach to polymer solar cells using one coating/printing machine, flexible substrates, no ITO, no vacuum and no spincoating[J]", Solar Energy Materials and Solar Cells, vol. 108, (2013), pp. 126-128.

[14] N. Espinosa, R. Garcia-Valverde and A. Urbina, "A life cycle analysis of polymer solar cell modules prepared using roll-to-roll methods under ambient conditions[J]", Solar Energy Materials and Solar Cells, vol. 95, no. 5, (2011), pp. 1293-1302.

[15] S. Nicolay, M. Benkhaira and L. Ding, "Control of CVD-deposited ZnO films properties through water/DEZ ratio: Decoupling of electrode morphology and electrical characteristics[J]", Solar Energy Materials and Solar Cells, vol. 105, (2012), pp. 46-52.

[16] D. R. Kim, C. H. Lee and J. M. Weisse, "Shrinking and Growing: Grain Boundary Density Reduction for Efficient Polysilicon Thin-Film Solar Cells[J]", Nano letters, vol. 12, no. 12, (2012), pp.6485-6491.

[17] A. Llordés, G. Garcia and J. Gazquez, "Tunable near-infrared and visible-light transmittance in nanocrystal-in-glass composites[J]”, Nature, vol. 500, no.7462, (2013), pp.323-326. 
International Journal of Control and Automation

Vol. 9 No. 5 (2016)

\section{Authors}

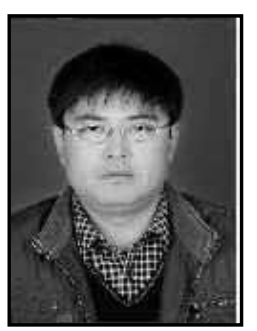

Junfu Qiao.Male, his Research interests : Application of automation technology, Automation and control technology in the areas of teaching and research work. 